Article

\title{
Diagnostic Performance of Risk of Malignancy Algorithm (ROMA), Risk of Malignancy Index (RMI) and Expert Ultrasound Assessment in a Pelvic Mass Classified as Inconclusive by International Ovarian Tumour Analysis (IOTA) Simple Rules
}

\author{
Siew Fei Ngu ${ }^{1}$, Yu Ka Chai ${ }^{2}$, Ka Man Choi ${ }^{3}$, Tsin Wah Leung ${ }^{4}$, Justin Li ${ }^{5}$ (D), Gladys S. T. Kwok ${ }^{1}$ (D), \\ Mandy M. Y. Chu ${ }^{1}$, Ka Yu Tse ${ }^{1}{ }^{(D}$, Vincent Y. T. Cheung ${ }^{1}$, Hextan Y. S. Ngan ${ }^{1}$ and Karen K. L. Chan ${ }^{1, * \mathbb{D}}$
}

check for

updates

Citation: Ngu, S.F.; Chai, Y.K.; Choi, K.M.; Leung, T.W.; Li, J.; Kwok, G.S.T.; Chu, M.M.Y.; Tse, K.Y.; Cheung, V.Y.T.; Ngan, H.Y.S.; et al. Diagnostic Performance of Risk of Malignancy Algorithm (ROMA), Risk of Malignancy Index (RMI) and Expert Ultrasound Assessment in a Pelvic Mass Classified as Inconclusive by International Ovarian Tumour Analysis (IOTA) Simple Rules. Cancers 2022, 14, 810. https://doi.org/10.3390/ cancers14030810

Academic Editor: Neville F. Hacker

Received: 9 January 2022

Accepted: 3 February 2022

Published: 5 February 2022

Publisher's Note: MDPI stays neutral with regard to jurisdictional claims in published maps and institutional affiliations.

Copyright: (C) 2022 by the authors. Licensee MDPI, Basel, Switzerland. This article is an open access article distributed under the terms and conditions of the Creative Commons Attribution (CC BY) license (https:// creativecommons.org/licenses/by/ $4.0 /)$.
1 Department of Obstetrics and Gynaecology, The University of Hong Kong, Queen Mary Hospital, Hong Kong, China; ngusiewf@hku.hk (S.F.N.); gstkwok@hku.hk (G.S.T.K.); chumy@hku.hk (M.M.Y.C.); tseky@hku.hk (K.Y.T.); vytc@hku.hk (V.Y.T.C.); hysngan@hku.hk (H.Y.S.N.)

2 Department of Obstetrics and Gynaecology, United Christian Hospital, Hong Kong, China; cyk095a@ha.org.hk

3 Department of Obstetrics and Gynaecology, Tseung Kwan O Hospital, Hong Kong, China; choikm@ha.org.hk 4 Department of Obstetrics and Gynaecology, Kwong Wah Hospital, Hong Kong, China; leungtw2@ha.org.hk 5 Li Ka Shing Faculty of Medicine, The University of Hong Kong, Hong Kong, China; just.li.328@gmail.com

* Correspondence: kklchan@hku.hk; Tel.: +852-2255-4518; Fax: +852-2855-0947

Simple Summary: The accurate prediction of malignancy for a pelvic mass detected on ultrasound allows for appropriate referral to specialised care. IOTA simple rules are one of the best methods but are inconclusive in $25 \%$ of cases, where subjective assessment by an expert sonographer is recommended but may not always be available. In the present paper, we evaluate the methods for assessing the nature of a pelvic mass, including IOTA with subjective assessment by expert ultrasound, RMI and ROMA. In particular, we investigate whether ROMA can replace expert ultrasound when IOTA is inconclusive. When IOTA was inconclusive, we found that expert ultrasound was more sensitive in diagnosing a malignant mass than ROMA, with no significant difference in the specificity or accuracy. All the assessment methods involving IOTA had similar accuracies, and they were more accurate than RMI or ROMA alone. Thus, IOTA should be the first step for assessing a pelvic mass. If inconclusive, an assessment by expert ultrasound is preferrable.

\begin{abstract}
The accurate prediction of malignancy for a pelvic mass detected on ultrasound allows for appropriate referral to specialised care. IOTA simple rules are one of the best methods but are inconclusive in $25 \%$ of cases, where subjective assessment by an expert sonographer is recommended but may not always be available. In the present paper, we evaluate the methods for assessing the nature of a pelvic mass, including IOTA with subjective assessment by expert ultrasound, RMI and ROMA. In particular, we investigate whether ROMA can replace expert ultrasound when IOTA is inconclusive. This prospective study involves one cancer centre and three general units. Women scheduled for an operation for a pelvic mass underwent a pelvic ultrasound pre-operatively. The final histology was obtained from the operative sample. The sensitivity, specificity and accuracy for each method were compared with the McNemar test. Of the 690 women included in the study, $171(25 \%)$ had an inconclusive IOTA. In this group, expert ultrasound was more sensitive in diagnosing a malignant mass compared to ROMA $(81 \%$ vs. $63 \%, p=0.009)$ with no significant difference in the specificity or accuracy. All assessment methods involving IOTA had similar accuracies and were more accurate than RMI or ROMA alone. In conclusion, when IOTA was inconclusive, assessment by expert ultrasound was more sensitive than ROMA, with similar specificity.
\end{abstract}


Keywords: pelvic mass; ovarian cancer; biomarkers; HE4; CA125; risk of malignancy index (RMI); risk of malignancy algorithm (ROMA); international ovarian tumour analysis simple rules (IOTA)

\section{Introduction}

Pelvic ultrasound is one of the most common investigations carried out for various gynaecological symptoms. Often, an ovarian or pelvic mass is found on ultrasound examination. The key question is whether the mass is benign or malignant. This has important implications because it determines the subsequent management. If the mass is likely to be benign, it can be observed or operated by general gynaecologists, usually laparoscopically. However, if it is malignant, the management would be very different. The patient would need to be referred to a gynaecological oncologist, as it has been shown that ovarian cancers managed by gynaecological oncologists would have better outcomes [1,2]. Therefore, the accurate determination of the likelihood of malignancy before the operation is important to avoid delaying the management of ovarian cancer, leading to a poorer prognosis. On the other hand, there would be unnecessary interventions and operative procedures with associated additional risks if a benign mass is managed as if it were malignant. Unlike other malignancies for which a biopsy can be taken to determine the nature of a lesion, a biopsy cannot be taken from an ovarian cyst as it would rupture the cyst, with the spillage of cyst contents into the abdomen, potentially leading to acute abdomen and up-staging of the cancer if the cyst turns out to be malignant. Therefore, methods for predicting the nature of the mass are needed.

Different methods have been developed to predict the likelihood of malignancy in a pelvic mass found on ultrasound [3-6]. One of the commonly used methods is the Risk of Malignancy Index (RMI), which is a product of the ultrasound morphological features, menopausal status and serum tumour marker CA125 [7-9]. This has been recommended by the Royal College of Obstetricians and Gynaecologists (U.K.) guidelines [10]. RMI has a pooled sensitivity of $78 \%$ (95\% confidence interval, CI $71-85 \%$ ) and specificity of $87 \%$ (95\% CI 83-91\%) [11].

The Risk of Malignancy Algorithm (ROMA) was developed using two tumour markers: CA125 and human epididymis protein 4 (HE4). CA125 is the most widely used tumour marker for ovarian cancer. However, CA125 is also raised in many conditions common in pre-menopausal women, such as fibroids, endometriosis and pelvic infection, and it is only raised in $50 \%$ of early-stage ovarian cancer [12]. Among other markers, HE4 has been the most promising [13]. HE4 was found to be elevated in more than half of ovarian tumours that do not express CA125 [14]. Therefore, a dual marker algorithm combining HE4 and CA125 was developed [15,16]. ROMA had similar sensitivity to CA125, but improved specificity, especially in pre-menopausal women. In addition, ROMA had an $83 \%$ accuracy in diagnosing early-stage disease [17]. In a meta-analysis comparing HE4, CA125 and ROMA, HE4 was found to be more useful in pre-menopausal women, while CA125 and ROMA were better in postmenopausal women [18]. The idea of combining ultrasound features and ROMA or HE4 was also investigated [19]. Combining ultrasound with HE4 can improve the sensitivity for detecting ovarian cancer compared to other algorithms. However, this has the same disadvantage in that it requires detailed ultrasound features, which are subject to variable interpretation, and therefore preclude its use in common clinical practice.

The International Ovarian Tumour Analysis (IOTA) study group developed a set of simple ultrasound-based rules $[20,21]$ with a reported sensitivity of $92 \%$ and specificity of $96 \%$ [22-26]. Patients whose masses could not be classified with these rules would be referred for a subjective expert ultrasound assessment. With a combination of simple rules triage, followed by subjective expert ultrasound in those with inconclusive results, a sensitivity of $91-93 \%$ and specificity of $93 \%$ could be achieved. In a recent meta-analysis of 
19 studies, IOTA simple rules were superior to all other methods, with an overall sensitivity of $93 \%$ and specificity of $81 \%$ [27].

IOTA simple rules seem to be a good option, but the results are inconclusive in $25 \%$ of the patients [20]. In this $25 \%$ of patients, an expert ultrasound is necessary. Unfortunately, the expertise of experienced ultrasound examiners in pelvic mass assessment is not easily transferred to less experienced examiners. Such expertise may not always be available. Therefore, we should have a strategy as an alternative if an expert is unavailable. With HE4 being more promising than CA125, especially in pre-menopausal women and earlystage disease, we evaluated the diagnostic performance of ROMA combined with IOTA simple rules. This study aims to assess whether ROMA could replace the need for assessment by experts for those women with inconclusive results from the IOTA simple rules.

\section{Materials and Methods}

\subsection{Study Design}

This was a multicentre prospective cohort study involving women scheduled for operation for a pelvic mass at the Department of Obstetrics and Gynaecology from one cancer centre (Queen Mary Hospital) and three general hospitals (United Christian Hospital, Pamela Youde Nethersole Hospital and Kwong Wah Hospital). The study was approved by the institutional review boards at each site and was registered at the HKU Clinical Trials Registry.

The primary research question was: "Among women with an adnexal mass scheduled for surgery who have inconclusive IOTA results, could ROMA predict malignancy better than assessment by expert ultrasound?". We hypothesised that, in this group of women, the additional use of ROMA would achieve similar accuracy of prediction compared to referring these women for expert ultrasound assessments.

The secondary research questions were: "1. Among women with an adnexal mass scheduled for surgery who have conclusive IOTA results, which method (IOTA, ROMA or RMI) was the best at predicting ovarian malignancy?"; "2. Which method was the best at predicting ovarian malignancy among all women with ovarian pathology?" We compared the accuracy of IOTA simple rules followed by an expert ultrasound or ROMA, or RMI with ROMA alone, or RMI alone; "3. Does the performance of these tests vary by menopausal status (pre vs. postmenopausal), hospital settings (cancer specialist centre vs. general hospitals) or tumour histology or stage?"

Women over the age of 18 who were found to have a pelvic mass on ultrasound, magnetic resonance imaging, computed tomography or positron emission tomography scan and were scheduled for operation, including ovarian cystectomy and oophorectomy (laparoscopic or open), were eligible for the study and were recruited consecutively. The women who declined a transvaginal ultrasound scan, were pregnant, with a previous history of ovarian, peritoneal or fallopian tube cancer or unknown malignancy, and history of bilateral oophorectomy were excluded. Those women for whom surgical removal was delayed for more than 120 days from the date of the ultrasound examination were also excluded. All the participants were informed of the study and provided written consent.

The participants underwent an ultrasound assessment pre-operatively by gynaecologists with basic ultrasound training, who were not experts in ultrasounds. A small group of gynaecologists at each hospital participated in the scans for this study. Each scan was performed by one gynaecologist and he/she classified the patients into high risk or low risk for malignancy according to the IOTA simple rules and RMI (Table 1). Ultrasound machines with transvaginal probe frequencies ranging from $5-12 \mathrm{MHz}$ were used. Greyscale and colour Doppler images were obtained for the morphology and blood flow. A transabdominal ultrasound was used for large masses that could not be completely visualised by transvaginal scan. The dominant mass was selected for analysis in women with multiple masses. During the ultrasound assessment, information on the following variables was collected, as suggested by the original IOTA papers $[10,20]$. The IOTA simple rules are based on five ultrasound features of malignancy (M-features) and five ultrasound features of benign lesion (B-features). The M-features are irregular solid tumour, presence of ascites, 
at least four papillary structures, irregular multilocular solid tumour $\geq 100 \mathrm{~mm}$ in diameter and very strong blood flow (IOTA colour score 4 ). The B-features are unilocular cysts, the presence of solid components of which the largest solid component $<7 \mathrm{~mm}$ in diameter, acoustic shadows, smooth multilocular tumour $<100 \mathrm{~mm}$ in diameter and no blood flow (IOTA colour score 1). The mass was classified as malignant if one or more M-features were present in the absence of a B-feature. The mass was classified as benign if one or more B-features were present in the absence of an M-feature. If both M-features and B-features were present or none of the features were present, the simple rules would be inconclusive. Those with inconclusive IOTA results underwent an expert ultrasound assessment by an expert sonographer or a gynaecologist who had obtained accreditation in Obstetric and Gynaecological Ultrasonography (a local competency-based assessment) and had been in practice in gynaecological ultrasound for more than five years. Ultrasound features required for RMI assessment, such as multilocular cysts, solid areas, metastases, ascites and bilateral lesions, were also recorded. The ROMA and RMI were calculated according to the suggestion by Moore and Jacobs, respectively $[7,15]$. The summary of the assessment methods used in predicting malignancy in a pelvic mass found on ultrasound investigated in this study is shown in Table 1.

Table 1. Methods used in predicting malignancy in a pelvic mass found on ultrasound investigated in this study.

\begin{tabular}{|c|c|c|c|}
\hline \multirow{2}{*}{ Methods } & \multirow{2}{*}{ Components } & \multicolumn{2}{|c|}{ Risk of Malignancy } \\
\hline & & High Risk & Low Risk \\
\hline IOTA simple rules (IOTA) & $\begin{array}{l}\text { Ultrasound assessment using } \\
5 \text { benign (B-features) and } \\
5 \text { malignant features (M-features) }\end{array}$ & $\begin{array}{l}\text { Presence of }>1 \mathrm{M} \text {-features } \\
\text { and absence of B-features }\end{array}$ & $\begin{array}{l}\text { Presence of }>1 \text { B-features } \\
\text { and absence of } \mathrm{M} \text {-features }\end{array}$ \\
\hline $\begin{array}{l}\text { Risk of malignancy } \\
\text { algorithm (ROMA) }\end{array}$ & $\begin{array}{l}\text { Calculation of risk by an algorithm } \\
\text { taking into account the menopausal } \\
\text { status, CA125 and HE4 levels } \\
\text { Premenopausal Predictive Index } \\
(\mathrm{PI})=-12.0+2.38 \times \mathrm{LN} \text { (natural } \\
\text { log) }[\mathrm{HE} 4]+0.0626 \times \mathrm{LN}[\mathrm{CA} 125] \\
\text { Postmenopausal PI }=-8.09+1.04 \\
\times \mathrm{LN}[\mathrm{HE} 4]+0.732 \times \mathrm{LN}[\mathrm{CA} 125] \\
\text { ROMA }=\exp (\mathrm{PI}) /[1+\exp (\mathrm{PI})] \times 100\end{array}$ & $\begin{array}{l}\text { Premenopausal: } \\
\text { ROMA } \geq 7.4 \\
\text { Postmenopausal: } \\
\text { ROMA } \geq 25.3\end{array}$ & $\begin{array}{l}\text { Premenopausal: } \\
\text { ROMA < } 7.4 \\
\text { Postmenopausal: } \\
\text { ROMA }<25.3\end{array}$ \\
\hline $\begin{array}{l}\text { Risk of malignancy index } \\
\text { (RMI) }\end{array}$ & $\begin{array}{l}\text { Calculation of risk by ultrasound } \\
\text { score }(\mathrm{U}) \text {, menopausal status }(\mathrm{M}) \\
\text { and } \mathrm{CA} 125 \text { level } \\
\mathrm{RMI}=\mathrm{U} \times \mathrm{M} \times \mathrm{CA} 125\end{array}$ & $\mathrm{RMI} \geq 200$ & $\mathrm{RMI}<200$ \\
\hline Expert ultrasound & $\begin{array}{l}\text { Subjective assessment by expert } \\
\text { sonographer }\end{array}$ & $\begin{array}{l}\text { Assessment suggestive of } \\
\text { malignancy }\end{array}$ & $\begin{array}{l}\text { Assessment suggestive of } \\
\text { benign tumour }\end{array}$ \\
\hline
\end{tabular}

Blood was taken for the CA125 and HE4 levels pre-operatively. A total of $10 \mathrm{~mL}$ of blood was collected into a serum or serum separator tube, centrifuged, aliquoted and stored at $-20^{\circ} \mathrm{C}$ or colder within $4 \mathrm{~h}$. Blood samples taken from the three general hospitals were transported on dry ice to the cancer centre on the same day for central analysis. Samples were tested using the ARCHITECT CA125 II assay and ARCHITECT HE4 assay (Abbott Diagnostics, Abbott Park, IL, USA), according to the manufacturer's instructions.

Surgery was performed by laparotomy or laparoscopy based on the surgeon's decision. Excised tumour tissues were histologically examined at the local hospital. The histological assessment was performed by the pathologist without the knowledge of the ultrasound results.

For each woman, five prediction parameters, including (1) IOTA with expert ultrasound, (2) IOTA with ROMA, (3) IOTA with RMI, (4) ROMA alone and (5) RMI alone were calculated, and the results were correlated with the histology results: benign or malignant (including borderline tumours) from the surgery. 


\subsection{Sample Size Calculation}

Our study focused on the group of women with inconclusive results from IOTA simple rules. Previous studies showed that this group represented about $25 \%$ of women undergoing IOTA assessment [20]. We assumed that the accuracy rate for ROMA was around $85 \%$, and the actual difference between the 2 methods was 5\% $[17,20]$. The range of non-inferiority was assumed at 5\%. A sample size of 160 women would achieve $90 \%$ power to show non-inferiority between the 2 correlated accuracy rates. The calculation used a one-sided non-inferiority test of two correlated proportions. To achieve 160 women, a minimum of 640 women undergoing the operation would be needed.

\subsection{Statistical Analysis}

Data were analysed with the help of the expertise in the Biostatistics and Clinical Research Methodology Unit at the School of Public Health at the University of Hong Kong. The primary analysis focused on evaluating the sensitivity, specificity and accuracy of ROMA and assessment by expert ultrasound among women with inconclusive IOTA results. Sensitivity, specificity and accuracy (defined as the number of correct assessments divided by the number of all assessments) were compared using the McNemar test. Similar analyses were repeated for the secondary outcomes. Subgroup analyses were performed for different menopausal status, hospitals and histological subtypes. The $95 \%$ confidence interval of sensitivity, specificity and accuracy was calculated. The analyses were conducted using $\mathrm{R}$ version 4.1.0 (R Foundation for Statistical Computing Platform), IBM SPSS Statistics Version 25 or the link: https: / / www2.ccrb.cuhk.edu.hk/stat/confidence\%20interval/McNemar\%20Test.htm (accessed on 31 August 2021). $p<0.05$ is considered statistically significant.

\section{Results}

\subsection{Study Population}

Between 30 April 2018 and 10 August 2020, 814 women were recruited: 408 were from a cancer centre, and 406 were from the general hospitals. A total of 678 women completed all study procedures, including the ultrasound, blood tests for CA125 and HE4 and surgery for the pelvic mass. An additional 19 women did not undergo surgery, but the diagnosis of malignancy was made on biopsy or cytology. Overall, 690 women had a histological or cytological diagnosis, and they were included in the final analysis (Figure 1). The median age was 46 years (range 18-69). The proportion of malignant pelvic masses was higher at the cancer centre than in the general hospitals (32.8\% vs. 8.6\%). The background demographic, histology and stage of the disease for the whole study population and those with inconclusive IOTA results are shown in Table $2 a, b$. 


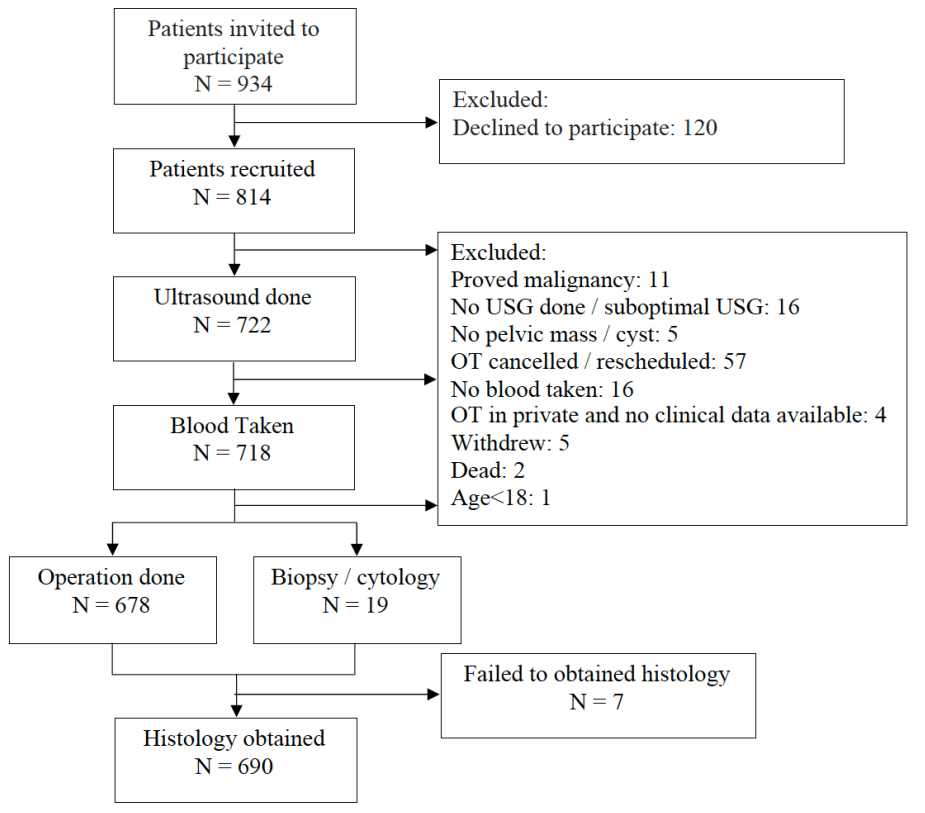

Figure 1. The flow of participants. 
Table 2. (a) Demographics for the whole study population. (b) Demographics for women with inconclusive IOTA simple rules results.

\begin{tabular}{|c|c|c|c|}
\hline \multicolumn{4}{|c|}{ (a) } \\
\hline & Cancer Centre & General Units & Total \\
\hline No. of patients & $341(49.4 \%) *$ & $349(50.6 \%) *$ & $690(100 \%) *$ \\
\hline Age (median) & $47(18-85)$ & $45(19-89)$ & $46(18-89)$ \\
\hline \multicolumn{4}{|l|}{ Menopausal status } \\
\hline Postmenopausal & $113(33.1 \%)$ & $99(28.4 \%)$ & $212(30.7 \%)$ \\
\hline Pre-menopausal & $228(66.9 \%)$ & $250(71.6 \%)$ & $478(69.3 \%)$ \\
\hline No. of ovarian malignancies (\%) & $112(32.8 \%)$ & $30(8.6 \%)$ & $142(20.6 \%)$ \\
\hline \multicolumn{4}{|l|}{ Histology } \\
\hline \multicolumn{4}{|l|}{ Ovarian } \\
\hline Benign & $184(54.0 \%)$ & $275(78.8 \%)$ & $459(66.5 \%)$ \\
\hline Endometriotic cyst & $84(45.7 \%)$ & $97(35.3 \%)$ & $181(39.4 \%)$ \\
\hline Dermoid & $32(17.4 \%)$ & $7125.8 \%)$ & $103(22.4 \%)$ \\
\hline Serous/mucinous cystadenoma & $34(18.5 \%)$ & $57(20.7 \%)$ & $91(19.8 \%)$ \\
\hline Fibroma & $5(2.7 \%)$ & $7(2.5 \%)$ & $12(2.6 \%)$ \\
\hline Functional cyst & $6(3.3 \%)$ & $8(2.9 \%)$ & $14(3.1 \%)$ \\
\hline Hydrosalpinx & $1(0.5 \%)$ & $2(0.7 \%)$ & $3(0.7 \%)$ \\
\hline Mixed & $3(1.6 \%)$ & $1(0.4 \%)$ & $4(0.9 \%)$ \\
\hline Others/unspecified & $19(10.3 \%)$ & $32(11.6 \%)$ & $51(11.1 \%)$ \\
\hline Malignant & $112(32.8 \%)$ & $30(8.6 \%)$ & $142(20.6 \%)$ \\
\hline Serous & $28(25.0 \%)$ & $9(30.0 \%)$ & $37(26.1 \%)$ \\
\hline Mucinous & $5(4.5 \%)$ & $2(6.7 \%)$ & $7(4.9 \%)$ \\
\hline Clear cell & $25(22.3 \%)$ & $5(16.7 \%)$ & $30(21.1 \%)$ \\
\hline Endometrioid & $18(16.1 \%)$ & $7(23.3 \%)$ & $25(17.6 \%)$ \\
\hline Mixed & $13(11.6 \%)$ & $0(0 \%)$ & $13(9.2 \%)$ \\
\hline Sex cord stromal/germ cell & $4(3.6 \%)$ & $2(6.7 \%)$ & $6(4.2 \%)$ \\
\hline Metastatic & $10(8.9 \%)$ & $4(13.3 \%)$ & $14(9.9 \%)$ \\
\hline Others & $9(8.0 \%)$ & $1(3.3 \%)$ & $10(7.0 \%)$ \\
\hline Borderline & $16(4.7 \%)$ & $20(5.7 \%)$ & $36(5.2 \%)$ \\
\hline Malignant/borderline & $1(0.3 \%)$ & $0(0 \%)$ & $1(0.1 \%)$ \\
\hline \multicolumn{4}{|l|}{ Non-ovarian } \\
\hline Benign & $10(2.9 \%)$ & $23(6.6 \%)$ & $33(4.8 \%)$ \\
\hline Malignant & $18(5.3 \%)$ & $1(0.3 \%)$ & $19(2.8 \%)$ \\
\hline \multicolumn{4}{|l|}{ FIGO staging } \\
\hline 0 & $36(39.6 \%)$ & $15(65.2 \%)$ & $51(44.7 \%)$ \\
\hline II & $11(12.1 \%)$ & $4(17.4 \%)$ & $15(13.2 \%)$ \\
\hline III & $23(25.3 \%)$ & $2(8.7 \%)$ & $25(21.9 \%)$ \\
\hline IV & $10(11.0 \%)$ & $2(8.7 \%)$ & $12(10.5 \%)$ \\
\hline Unstaged & $11(12.1 \%)$ & $0(0 \%)$ & $11(9.6 \%)$ \\
\hline No. of inconclusive IOTA (\%) & $105(30.8 \%)$ & $66(18.9 \%)$ & $171(24.8 \%)$ \\
\hline
\end{tabular}


Table 2. Cont.

\begin{tabular}{|c|c|c|c|}
\hline \multicolumn{4}{|c|}{ (b) } \\
\hline & Cancer Centre & General Units & Total \\
\hline No. of patients & $105(61.4 \%) *$ & $66(38.6 \%) *$ & $171(100 \%) *$ \\
\hline Age (median) & $49(21-84)$ & $46.5(22-83)$ & $48(21-84)$ \\
\hline \multicolumn{4}{|l|}{ Menopausal status } \\
\hline Postmenopausal & $46(43.8 \%)$ & $21(31.8 \%)$ & $67(39.2 \%)$ \\
\hline Pre-menopausal & $59(56.2 \%)$ & $45(68.2 \%)$ & $104(60.8 \%)$ \\
\hline No. of ovarian malignancies (\%) & $46(43.8 \%)$ & $7(10.6 \%)$ & $53(31.0 \%)$ \\
\hline \multicolumn{4}{|l|}{ Histology } \\
\hline \multicolumn{4}{|l|}{ Ovarian } \\
\hline Benign & $37(35.2 \%)$ & $48(72.7 \%)$ & $85(49.7 \%)$ \\
\hline Endometriotic cyst & $11(29.7 \%)$ & $15(31.3 \%)$ & $26(30.6 \%)$ \\
\hline Dermoid & $7(18.9 \%)$ & $15(31.3 \%)$ & $22(25.9 \%)$ \\
\hline Serous/mucinous cystadenoma & $12(32.4 \%)$ & $8(16.7 \%)$ & $20(23.5 \%)$ \\
\hline Fibroma & $2(5.4 \%)$ & $5(10.4 \%)$ & $7(8.2 \%)$ \\
\hline Functional cyst & $1(2.7 \%)$ & $0(0 \%)$ & $1(1.2 \%)$ \\
\hline Others/unspecified & $4(10.8 \%)$ & $5(10.4 \%)$ & $9(10.6 \%)$ \\
\hline Malignant & $46(43.8 \%)$ & $7(10.6 \%)$ & $53(31.0 \%)$ \\
\hline Serous & $8(17.4 \%)$ & $1(1.5 \%)$ & $9(5.3 \%)$ \\
\hline Mucinous & $3(6.5 \%)$ & $0(0 \%)$ & $3(1.8 \%)$ \\
\hline Clear cell & $16(34.8 \%)$ & $1(1.5 \%)$ & $17(9.9 \%)$ \\
\hline Endometrioid & $4(8.7 \%)$ & $2(3.0 \%)$ & $6(3.5 \%)$ \\
\hline Mixed & $4(8.7 \%)$ & $0(0 \%)$ & $4(2.3 \%)$ \\
\hline Sex cord stromal/germ cell & $2(4.3 \%)$ & $1(1.5 \%)$ & $3(1.8 \%)$ \\
\hline Metastatic & $6(13.0 \%)$ & $1(1.5 \%)$ & $7(4.1 \%)$ \\
\hline Others & $3(6.5 \%)$ & $1(1.5 \%)$ & $4(2.3 \%)$ \\
\hline Borderline & $9(8.6 \%)$ & $7(10.6 \%)$ & $16(9.4 \%)$ \\
\hline Malignant/borderline & $1(1.0 \%)$ & $0(0 \%)$ & $1(0.6 \%)$ \\
\hline \multicolumn{4}{|l|}{ Non-ovarian } \\
\hline Benign & $4(3.8 \%)$ & $3(4.5 \%)$ & $7(4.1 \%)$ \\
\hline Malignant & $8(7.6 \%)$ & $1(1.5 \%)$ & $9(5.3 \%)$ \\
\hline \multicolumn{4}{|l|}{ FIGO staging } \\
\hline 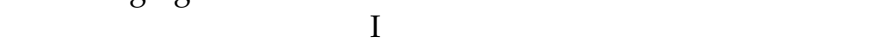 & $17(43.6 \%)$ & $3(50.0 \%)$ & $20(44.4 \%)$ \\
\hline II & $2(5.1 \%)$ & $2(33.3 \%)$ & $4(8.9 \%)$ \\
\hline III & $12(30.8 \%)$ & $1(16.7 \%)$ & $13(28.9 \%)$ \\
\hline IV & $2(5.1 \%)$ & $0(0 \%)$ & $2(4.4 \%)$ \\
\hline Unstaged & $6(15.4 \%)$ & $0(0 \%)$ & $6(13.3 \%)$ \\
\hline
\end{tabular}

${ }^{*}$ Row percentages, all others are column percentages.

\subsection{Amongst Women with an Inconclusive Result from the IOTA Simple Rules}

We compared the accuracy of the two methods, the expert ultrasound versus ROMA in the group of women who had inconclusive IOTA results ( $n=171,25 \%$ ) (Table 3 ). Expert ultrasound was more sensitive in diagnosing a malignant mass than ROMA (81\% vs. $63 \%, p=0.009)$, with no significant difference in the specificity and accuracy. For the IOTA with expert ultrasound, 15 out of 79 malignant tumours (19\%) were wrongly predicted to be benign, while 29 (37\%) were incorrectly predicted to be benign by IOTA with ROMA $(p=0.009)$. On the other hand, IOTA with expert ultrasound and IOTA with ROMA wrongly classified similar proportions of patients as malignant when the final pathology turned out to be benign ( $28 \%$ vs. $27 \%)$, respectively. 
Table 3. (a) Correlation of the diagnostic tests results with the histology results in women with inconclusive IOTA results $(n=171)$. (b) Sensitivity, specificity and accuracy for expert ultrasound and ROMA in women with inconclusive IOTA results $(\mathrm{n}=171)$.

\begin{tabular}{|c|c|c|c|}
\hline \multicolumn{4}{|c|}{ (a) } \\
\hline & \multirow{2}{*}{ Risk of Malignancy } & \multicolumn{2}{|c|}{ Histology } \\
\hline & & Malignant & Benign \\
\hline \multirow[t]{2}{*}{ Expert ultrasound } & High risk & 64 & 26 \\
\hline & Low risk & 15 & 66 \\
\hline \multirow[t]{4}{*}{ ROMA } & High risk & 50 & 25 \\
\hline & Low risk & 29 & 67 \\
\hline & \multicolumn{2}{|c|}{ (b) } & \\
\hline & Sensitivity $(95 \% \mathrm{CI})$ & Specificity $(95 \%$ CI $)$ & Accuracy $(95 \% \mathrm{CI})$ \\
\hline Expert ultrasound & $81.0 \%(70.3-88.6 \%)$ & $71.7 \%(61.2-80.4 \%)$ & $76.0 \%(68.8-82.1 \%)$ \\
\hline ROMA & $63.3 \%(51.6-73.6 \%)$ & $72.8 \%(62.4-81.3 \%)$ & $68.4 \%(60.8-75.2 \%)$ \\
\hline
\end{tabular}

\subsection{Amongst Women with a Conclusive Result from the IOTA Simple Rules}

Of the 690 women with a histological or cytological diagnosis, the IOTA simple rules were conclusive in 519 women (75\%). Amongst these women, IOTA and ROMA had similar sensitivities ( $81 \%$ and $82 \%$, respectively), and both had better sensitivities than RMI (71\%, $p=0.019$ and $p=0.006$, respectively). However, RMI had a better specificity than ROMA (94\% vs. $85 \%, p<0.001)$. Overall, IOTA was more accurate than ROMA or RMI in diagnosing a malignant mass (94\% vs. $84 \%$ and $89 \%, p \leq 0.001)$. Therefore, among all the women with conclusive IOTA results, IOTA is better at differentiating the malignant and benign cases correctly compared to ROMA or RMI (Table 4).

Table 4. (a) Correlation of the diagnostic tests with the histology results in women with conclusive IOTA results $(n=519)$. (b) Sensitivity, specificity and accuracy for IOTA, ROMA and RMI in women with conclusive IOTA results $(\mathrm{n}=519)$.

(a)

\begin{tabular}{cccc}
\hline \multirow{2}{*}{ Risk of Malignancy } & \multicolumn{2}{c}{ Histology } \\
\cline { 3 - 4 } & & Malignant & Benign \\
\hline \multirow{2}{*}{ IOTA } & High risk & 96 & 10 \\
& Low risk & 23 & 390 \\
\hline \multirow{2}{*}{ ROMA } & High risk & 97 & 59 \\
& Low risk & 22 & 341 \\
\hline \multirow{2}{*}{ RMI } & High risk & 84 & 23 \\
& Low risk & 35 & 377
\end{tabular}

(b)

\begin{tabular}{|c|c|c|c|}
\hline & Sensitivity $(95 \% \mathrm{CI})$ & Specificity $(95 \%$ CI $)$ & Accuracy $(95 \% \mathrm{CI})$ \\
\hline IOTA & $80.7 \%(72.2-87.1 \%)$ & $97.5 \%(95.3-98.7 \%)$ & $93.6 \%$ (91.1-95.5\%) \\
\hline ROMA & $81.5 \%(73.1-87.8 \%)$ & $85.3 \%(81.3-88.5 \%)$ & $84.4 \%(80.9-87.3 \%)$ \\
\hline RMI & $70.6 \%(61.4-78.4 \%)$ & $94.3 \%(91.4-96.2 \%)$ & $88.8 \%$ (85.7-91.3\%) \\
\hline
\end{tabular}

\subsection{Amongst the Whole Population of Women with an Ovarian Pathology}

We then explored which method best predicted ovarian malignancy for the whole study population (Table 5). Out of 640 women with an ovarian pathology, the IOTA with expert ultrasound was more sensitive than IOTA with ROMA or RMI ( $p=0.015$ and $p=0.001$, respectively). ROMA alone was more sensitive than RMI alone ( $p=0.007)$. The addition of IOTA to ROMA did not improve the sensitivity. All methods involving IOTA had similar 
specificities. RMI alone was more specific than ROMA alone $(p<0.001)$. The addition of IOTA to RMI further improved the specificity of RMI from 91\% to 94\% ( $p=0.003$ ).

Table 5. Diagnostic accuracy for five different strategies in all women with an ovarian pathology $(\mathrm{n}=640)$.

\begin{tabular}{cccl}
\hline & Sensitivity (95\% CI) & Specificity (95\% CI) & Accuracy (95\%CI) \\
\hline IOTA + expert & $79.9 \%(73.1-85.3 \%)$ & $92.8 \%(90.0-94.9 \%)$ & $89.2 \%(86.5-91.5 \%)$ \\
IOTA + ROMA & $73.2 \%(66.0-79.4 \%)$ & $93.7 \%(91.0-95.7 \%)$ & $88.0 \%(85.1-90.3 \%)$ \\
IOTA + RMI & $72.1 \%(64.8-78.4 \%)$ & $94.1 \%(91.5-96.0 \%)$ & $88.0 \%(85.1-90.3 \%)$ \\
ROMA alone & $74.3 \%(67.1-80.4 \%)$ & $84.4 \%(80.7-87.5 \%)$ & $81.6 \%(78.3-84.4 \%)$ \\
RMI alone & $66.5 \%(59.0-73.2 \%)$ & $91.1 \%(88.0-93.5 \%)$ & $84.2 \%(81.1-86.9 \%)$ \\
\hline
\end{tabular}

In terms of accuracy, both IOTA with ROMA and IOTA with RMI were similar to IOTA with expert ultrasound. RMI alone was more accurate than ROMA alone $(p=0.054)$. The combination of IOTA with expert ultrasound, ROMA or RMI were all more accurate than ROMA or RMI alone (IOTA with expert vs. ROMA alone $89 \%$ vs. 82\%, $p<0.001$; IOTA with expert vs. RMI alone $89 \%$ vs. $84 \%, p=0.001$; IOTA with ROMA vs. ROMA alone $88 \%$ vs. $82 \%$, $p<0.001$; IOTA with ROMA vs. RMI alone $88 \%$ vs. $84 \%, p=0.004$; IOTA with RMI vs. ROMA alone $88 \%$ vs. $82 \%, p<0.001$; and IOTA with RMI vs. RMI alone $88 \%$ vs. $84 \%, p<0.001$ ).

\subsection{Performance in Pre- and Postmenopausal Women}

In pre-menopausal women, similar to the overall population, IOTA with expert ultrasound is more sensitive than IOTA with ROMA ( $81 \%$ vs. $73 \%, p=0.035)$ (Table 6). ROMA alone was more sensitive than RMI alone (76\% vs. 66\%, $p=0.013)$. Adding IOTA to ROMA or RMI did not improve the sensitivity of either test alone. In terms of specificity, IOTA with expert ultrasound was similar to IOTA with ROMA (94\% vs. 94\%). RMI alone was more specific than ROMA alone $(92 \%$ vs. $84 \%, p<0.005)$. The addition of IOTA improved the specificity of RMI or ROMA alone ( $p<0.005$ for both). All strategies with IOTA had similar accuracies (IOTA with expert ultrasound vs. IOTA with RMI, $p=0.500$; IOTA with expert ultrasound vs. IOTA with ROMA, $p=0.196$; and IOTA with ROMA vs. IOTA with RMI, $p=0.166$ ) and were more accurate than either ROMA or RMI alone (ROMA vs. IOTA with expert ultrasound, $p=0.00003$; ROMA vs. IOTA with ROMA, $p=0.00001$; ROMA vs. IOTA with RMI, $p=0.00001$; RMI vs. IOTA with expert ultrasound, $p=0.011$; RMI vs. IOTA with ROMA, $p=0.040$; and RMI vs. IOTA with RMI, $p=0.001)$. RMI was more accurate than ROMA $(p=0.010)$.

Table 6. Sensitivity, specificity and accuracy for pre- and postmenopausal women.

\begin{tabular}{cccc}
\hline & Sensitivity (95\% CI) & Specificity $\mathbf{( 9 5 \% ~ C I )}$ & Accuracy (95\%CI) \\
\hline Premenopausal & & & \\
\hline IOTA + expert & $80.9 \%(70.9-88.2 \%)$ & $94.1 \%(91.0-96.2 \%)$ & $91.5 \%(88.4-93.8 \%)$ \\
IOTA + ROMA & $73.0 \%(62.4-81.6 \%)$ & $94.4 \%(91.3-96.4 \%)$ & $90.1 \%(86.9-92.6 \%)$ \\
IOTA + RMI & $71.9 \%(61.2-80.7 \%)$ & $96.1 \%(93.3-97.7 \%)$ & $91.2 \%(88.1-93.6 \%)$ \\
ROMA alone & $76.4 \%(66.0-84.5 \%)$ & $84.0 \%(79.7-87.6 \%)$ & $82.5 \%(78.5-85.8 \%)$ \\
RMI alone & $66.3 \%(55.4-75.8 \%)$ & $92.4 \%(89.0-94.9 \%)$ & $87.2 \%(83.6-90.1 \%)$ \\
\hline Postmenopausal & & & \\
\hline IOTA + expert & $78.9 \%(68.8-86.5 \%)$ & $88.6 \%(80.5-93.7 \%)$ & $84.1 \%(78.0-88.8 \%)$ \\
IOTA + ROMA & $73.3 \%(62.8-81.9 \%)$ & $91.4 \%(83.9-95.8 \%)$ & $83.1 \%(76.9-87.9 \%)$ \\
IOTA + RMI & $72.2 \%(61.6-80.9 \%)$ & $87.6 \%(79.4-93.0 \%)$ & $80.5 \%(74.1-85.7 \%)$ \\
ROMA alone & $72.2 \%(61.6-80.9 \%)$ & $85.7 \%(77.2-91.5 \%)$ & $79.5 \%(73.0-84.8 \%)$ \\
RMI alone & $66.7 \%(55.9-76.0 \%)$ & $86.7 \%(78.3-92.3 \%)$ & $77.4 \%(70.8-83.0 \%)$ \\
\hline
\end{tabular}

In postmenopausal women, all IOTA strategies had similar sensitivities (Table 6). Adding IOTA to either ROMA or RMI did not improve the sensitivity, but IOTA with expert ultrasound was more sensitive than RMI alone (79\% vs. 67\%, $p=0.008$ ). ROMA and RMI had similar sensitivities $(72 \%$ vs. $66 \%, p=0.150)$. All strategies involving IOTA 
had similar specificities. Similar to pre-menopausal women, all strategies with IOTA had similar accuracies. IOTA with ROMA was similar to ROMA alone (83\% vs. 80\%, $p>0.05)$, but more accurate than RMI alone (83\% vs. 77\%, $p=0.027)$. ROMA and RMI alone had similar accuracies ( $80 \%$ vs. $77 \%$ ).

IOTA with expert and IOTA with ROMA had similar sensitivities in pre- and postmenopausal women ( $81 \%$ vs. $79 \%, p>0.05)$, but both were more accurate in premenopausal women (92\% vs. $84 \%, p=0.009 ; 90 \%$ vs. $83 \%, p=0.017$, respectively). ROMA and RMI had similar sensitivities and specificities in pre- and postmenopausal women, but RMI was more accurate in pre-menopausal women than in postmenopausal women (87\% vs. $77 \%, p=0.003)$.

\subsection{Performance in a Cancer Centre vs. General Hospitals (for Patients with an Ovarian Pathology)}

In the cancer centre $(\mathrm{n}=315)$, IOTA with expert ultrasound is more sensitive, but less specific than IOTA with ROMA ( $83 \%$ vs. $76 \%, p=0.033$ and $87 \%$ vs. $91 \%, p=0.040$, respectively), with overall no difference in the accuracy (85\% vs. 85\%) (Table 7 ). ROMA and RMI had similar sensitivities, but RMI was more specific ( $87 \%$ vs. $80 \%)$ with an overall similar accuracy. In the general units $(n=325)$, there was no significant difference between IOTA with expert ultrasound and IOTA with ROMA in sensitivity, specificity and accuracy. IOTA with expert or ROMA, or ROMA alone, was more sensitive than RMI alone. The addition of IOTA to RMI improved the sensitivity of RMI alone (60\% vs. 42\%, $p=0.008$ ) and approximated that to ROMA alone. In both types of hospitals, the addition of IOTA improved the accuracy of ROMA or RMI alone.

Table 7. Diagnostic performance in the cancer unit vs. general units.

\begin{tabular}{|c|c|c|c|c|c|c|}
\hline & \multicolumn{2}{|c|}{ Sensitivity (95\% CI) } & \multicolumn{2}{|c|}{ Specificity $(95 \%$ CI $)$} & \multicolumn{2}{|c|}{ Accuracy $(95 \% \mathrm{CI})$} \\
\hline & Cancer & General & Cancer & General & Cancer & General \\
\hline IOTA + expert & $\begin{array}{c}82.9 \% \\
(75.1-88.8 \%)\end{array}$ & $\begin{array}{c}72.0 \% \\
(57.3-83.3 \%)\end{array}$ & $\begin{array}{c}87.1 \% \\
(81.2-91.4 \%)\end{array}$ & $\begin{array}{c}96.7 \% \\
(93.7-98.4 \%)\end{array}$ & $\begin{array}{c}85.4 \% \\
(80.9-89.0 \%)\end{array}$ & $\begin{array}{c}92.9 \% \\
(89.4-95.4 \%)\end{array}$ \\
\hline IOTA + ROMA & $\begin{array}{c}76.0 \% \\
(67.5-82.9 \%)\end{array}$ & $\begin{array}{c}66.0 \% \\
(51.1-78.4 \%)\end{array}$ & $\begin{array}{c}91.4 \% \\
(86.2-94.8 \%)\end{array}$ & $\begin{array}{c}95.3 \% \\
(91.9-97.4 \%)\end{array}$ & $\begin{array}{c}85.1 \% \\
(80.5-88.7 \%)\end{array}$ & $\begin{array}{c}90.8 \% \\
(87.0-93.6 \%)\end{array}$ \\
\hline IOTA + RMI & $\begin{array}{c}76.7 \% \\
(68.3-83.5 \%)\end{array}$ & $\begin{array}{c}60.0 \% \\
(45.2-73.3 \%) \\
\end{array}$ & $\begin{array}{c}91.9 \% \\
(86.8-95.3 \%) \\
\end{array}$ & $\begin{array}{c}95.6 \% \\
(92.3-97.6 \%) \\
\end{array}$ & $\begin{array}{c}85.7 \% \\
(81.2-89.3 \%) \\
\end{array}$ & $\begin{array}{c}90.2 \% \\
(86.3-93.1 \%) \\
\end{array}$ \\
\hline ROMA alone & $\begin{array}{c}79.8 \% \\
(71.7-86.2 \%)\end{array}$ & $\begin{array}{c}60.0 \% \\
(45.2-73.3 \%)\end{array}$ & $\begin{array}{c}79.6 \% \\
(72.9-85.0 \%)\end{array}$ & $\begin{array}{c}87.6 \% \\
(83.0-91.2 \%)\end{array}$ & $\begin{array}{c}79.7 \% \\
(74.7-83.9 \%)\end{array}$ & $\begin{array}{c}83.4 \% \\
(78.8-87.2 \%)\end{array}$ \\
\hline RMI alone & $\begin{array}{c}76.0 \% \\
(67.5-82.9 \%)\end{array}$ & $\begin{array}{c}42.0 \% \\
(28.5-56.7 \%)\end{array}$ & $\begin{array}{c}87.1 \% \\
(81.2-91.4 \%)\end{array}$ & $\begin{array}{c}93.8 \% \\
(90.1-96.2 \%)\end{array}$ & $\begin{array}{c}82.5 \% \\
(77.8-86.5 \%)\end{array}$ & $\begin{array}{c}85.8 \% \\
(81.5-89.4 \%)\end{array}$ \\
\hline
\end{tabular}

\subsection{Sensitivity in Diagnosing Early-Stage (Stage 1) Cancer}

There were 51 stage 1 cancers out of 142 ovarian cancers. The histology included 19 clear cell, 12 serous, 11 endometrioid, 6 mucinous, 1 seromucinous and 2 mixed clear cell and endometrioid. The sensitivity in diagnosing early-stage cancer for the different strategies is shown in Table 8. IOTA with expert ultrasound had a similar sensitivity compared with IOTA with ROMA/RMI or ROMA alone. All strategies involving IOTA were more sensitive than RMI alone (IOTA with expert $81 \%$ vs. RMI alone $58 \%, p=0.003$; IOTA with ROMA 72\% vs. RMI alone 58\%, $p=0.031$; and IOTA with RMI 70\% vs. RMI alone $58 \%, p=0.035)$. ROMA alone was more sensitive than RMI alone (70\% vs. 58\%), but this did not reach statistical significance $(p=0.061)$. IOTA with ROMA was not more accurate than IOTA with RMI (Table 8). 
Table 8. Sensitivity for predicting early-stage (stage 1) ovarian cancer.

\begin{tabular}{cc}
\hline & Sensitivity \\
\hline IOTA + expert & $80.7 \%(67.7-89.5 \%)$ \\
IOTA + ROMA & $71.9 \%(58.3-82.6 \%)$ \\
IOTA + RMI & $70.2 \%(56.4-81.2 \%)$ \\
ROMA alone & $70.2 \%(56.4-81.2 \%)$ \\
RMI alone & $57.9 \%(44.1-70.6 \%)$ \\
\hline
\end{tabular}

\subsection{Performance for Different Histological Types of Ovarian Cancer}

There were 36 borderline tumours included in the study. All strategies investigated in this study had a poorer sensitivity in diagnosing borderline tumours ranging from $36 \%$ to $57 \%$. There was no significant difference in the various methods. We also tried to explore the sensitivities for all non-epithelial tumours $(n=28)$. Again, the overall sensitivity was slightly lower than for epithelial tumours $(57-71 \%$ vs. $67-80 \%)$, but they did not reach statistical significance. This analysis was limited by the relative rarity of non-epithelial tumours.

\section{Discussion}

Many strategies and ultrasound criteria were investigated to predict malignancy in a pelvic mass detected on ultrasound. IOTA simple rules, ROMA and RMI were amongst the most commonly adopted methods, clinically. In this study, we compared the performances of these common strategies, particularly those for which the IOTA simple rules were inconclusive. The percentage of malignancy in our study (21\%) was in the same range as other published series (Table 9). Likewise, the percentage of malignancy among women with inconclusive IOTA in our study (31\%) was also comparable to other reported series ( $40 \%$ in Timmerman et al.) [20]. Similar to previous findings, IOTA, when yielding a conclusive result, was more accurate than ROMA and RMI. However, we found that IOTA was inconclusive in $25 \%$ of the women, similar to the percentage reported in the literature [20]. Subjective assessment by an expert sonographer was recommended in these cases with inconclusive IOTA results [20]. Nonetheless, there was limited data on how to assess this group of women if an expert was not available. Timmerman et al. reported the sensitivity of RMI or logistic regression in these cases, and they found that these were inferior to subjective assessment by an expert. The sensitivity reported was only $50 \%$ for RMI, while for subjective assessment it was $89 \%$ [20]. This was similar to our findings. The usefulness of ROMA in this situation is lacking. Potentially, with an additional tumour marker (HE4), it can out-perform RMI. It was reported that the addition of ROMA to expert subjective assessment did not further improve the diagnostic accuracy [28], but whether ROMA can replace subjective expert assessment was not reported. In this study, we explored the performance of ROMA in this group of patients. We found that ROMA was less sensitive than expert ultrasound. This suggested that expert ultrasound should be the investigation of choice when IOTA yielded inconclusive results. However, ROMA and RMI are possible options if expertise is not available since they have similar accuracy to expert ultrasound, despite a lower sensitivity. Since ROMA involves measuring an additional tumour marker, further considerations on the cost-effectiveness should be explored.

For the whole study population (IOTA conclusive and inconclusive), we investigated which strategy would be best at predicting ovarian cancer. Again, we found that IOTA with expert ultrasound was the most sensitive. All three strategies involving IOTA had similar accuracies and were more accurate than just ROMA or RMI alone. This suggested that we should adopt a strategy that involved IOTA as the first step in the assessment. If IOTA was inconclusive, we could use either expert assessment, ROMA or RMI to further predict the nature of the mass. We further explored if this applied to both cancer centres and general units where the prevalence of malignancy was very different. We found that the sensitivity of all the strategies was higher in the cancer centre, which was likely the result of a higher prevalence of malignancy in the cancer centre. Although a test's sensitivity is not expected 
to vary with disease prevalence, the fact that different sensitivities were observed suggested that the test may not function in the same way in the cancer centre and general units. The implications of a false positive or negative test would also be different in the cancer centre compared to the general units. In the general units, a missed diagnosis of malignancy (i.e., a low sensitivity) would lead to the need for a second comprehensive operation for the definitive cancer management after the simple surgery was performed at the general units. Meanwhile, an over-diagnosis of malignancy (i.e., low specificity) would lead to unnecessary referrals to the cancer centre for primary surgery. From the patient's view, it would be more important for the test to have a high sensitivity to avoid a second operation. Our study found that both IOTA with expert ultrasound and IOTA with ROMA offered the best sensitivity in the general units. However, we realised that expertise in ultrasound or ROMA might not be readily available at the general units. In this situation, adding IOTA to RMI (i.e., perform IOTA first, if inconclusive, use RMI) would be better than RMI alone since this would improve the sensitivity from $42 \%$ to $60 \%$.

Table 9. The percentage of malignancy, inconclusive IOTA, sensitivity and specificity in previous and present studies.

\begin{tabular}{|c|c|c|c|c|c|}
\hline & $\begin{array}{c}\text { Number of } \\
\text { Patients }\end{array}$ & $\begin{array}{c}\text { Malignancy } \\
\text { Prevalence \% }\end{array}$ & $\begin{array}{c}\text { Inconclusive } \\
\text { IOTA \% }\end{array}$ & Sensitivity $\%$ & Specificity $\%$ \\
\hline Timmerman D et al., 2010 [20] & 997 & 28 & 23 & 90 & 93 \\
\hline Hartman CA et al., 2012 [29] & 110 & 28 & 17 & 84 & 86 \\
\hline Alcazar JL et al., 2013 [30] & 340 & 16 & 21 & 89 & 96 \\
\hline Sayasneh A et al., 2013 [26] & 255 & 29 & 17 & 86 & 94 \\
\hline Nunes N et al., 2014 [23] & 303 & 44 & 22 & 94 & 89 \\
\hline $\begin{array}{c}\text { Ruiz de Gauna B et al., } 2015 \text { [25] } \\
\text { centre A }\end{array}$ & 114 & 27 & 18 & 100 & 89 \\
\hline $\begin{array}{l}\text { Ruiz de Gauna B et al., } 2015 \text { [25] } \\
\text { centre B }\end{array}$ & 133 & 11 & 18 & 86 & 88 \\
\hline Knafel A et al., 2013 [31] & 226 & NA & 18 & 95 & 74 \\
\hline Piovano E et al., 2017 [32] & 391 & 21 & 11 & 82 & 92 \\
\hline Current study & 640 & 21 & 25 & 80 & 93 \\
\hline
\end{tabular}

In a cancer centre, a missed diagnosis of malignancy might not lead to the need for a second operation because expertise would be available at the hospital. The gynaeoncologist could be called into the theatre to complete the full cancer operation when the frozen section of the mass showed malignancy, even though this would not be ideal logistically. On the other hand, if a benign mass was wrongly predicted to be malignant pre-operatively (i.e., low specificity), the woman may have undergone an unnecessary laparotomy with a midline incision rather than a minimally invasive procedure. In our centre, laparotomy is used in most patients with a suspected ovarian malignancy, although laparoscopic surgery may be considered in selected patient with isolated adnexal mass. From the patient's point of view, one might argue that a test with a high specificity would be more important in the cancer centre. Our results show that IOTA with ROMA is the most specific, and this might be the test of choice in a cancer centre. In situations where ROMA was unavailable, IOTA with expert ultrasound would be the second choice, as it was more specific than RMI alone.

We also investigated any differences in the performances of the various strategies in pre- and postmenopausal women. We found that IOTA strategies appeared to perform better in pre-menopausal women. This agrees with the subgroup analysis of a metaanalysis, which showed a higher accuracy of IOTA simple rules in pre-menopausal women, possibly due to better diagnosis of endometriotic or dermoid cysts [27]. Amongst the IOTA strategies, Timmerman et al. found that expert ultrasound was more sensitive than RMI in those with inconclusive results in both pre- and postmenopausal women [20]. Our study also had similar findings. In addition, we found that IOTA with expert ultrasound was also superior to IOTA with ROMA in pre-menopausal women, but not in postmenopausal 
women. Similar to the other reported series $[33,34]$, we found that RMI was more accurate in pre-menopausal women than in postmenopausal women, whereas ROMA had a similar accuracy in both pre and postmenopausal women.

We noted that RMI alone had a particularly low sensitivity for detecting early disease. This was expected since CA125, the tumour marker included in the calculation of RMI, is well known to be elevated in late but not early disease. Adding IOTA to RMI would improve the sensitivity from $58 \%$ to $70 \%$. The IOTA algorithm may have a role in ovarian cancer screening.

\section{Strengths and Weaknesses}

A strength of this study was its prospective multicentre design, involving both cancer centre and general units. This allows the results to be applicable in various settings with different prevalences of malignancy. Another strength was the availability of the reference standard (histological diagnosis) in nearly all the cases. In this study, assessments by IOTA were conducted by gynaecologists who were not ultrasound experts. This reflects the original purpose of IOTA for triaging patients in the general population for referral to specialised ultrasound experts. Our study results, therefore, can reflect realistic clinical scenarios. On the other hand, our study included patients who were already scheduled for surgery, and thus represented a selected group of women. In clinical practice, apart from deciding for the need for referral to the gynaecological oncologist, another dilemma would be whether a patient needs a surgery or not. Thus, our results on these different strategies may not be useful in informing the decision for surgery. Another limitation was that the gynaecologists conducting the IOTA assessment in this study did not attend any specific training courses in using IOTA. This may have affected the accuracy of IOTA and can explain the lower sensitivity in our population compared to those reported in the literature. Another limitation of the study was the different definition of the "expert" performing the expert ultrasound assessment in this study, compared to published training requirements. Internationally published training requirements for an expert ultrasound examiner specified that the expert should spend most of the time performing ultrasound examinations [35]. In our study, the experts were defined as gynaecologists with more than five years of ultrasound experience. These different definitions may affect the overall accuracy of the diagnostic tests in this study. Nonetheless, despite the different definitions of "experts", the superiority of expert ultrasound was still demonstrated in this study.

Similar to the other studies, our study evaluated the diagnostic performance of some of the more commonly used methods to predict the risk of malignancy of an adnexal mass. However, these studies did not consider some relevant clinical factors that may affect management, many of which would make these strategies inappropriate from practical perspective. For example, if a woman has a computed tomography that is suspicious for carcinomatosis, her surgery will be performed by a gynaecologic oncologist. Hence, the results of the IOTA, RMI or ROMA or any combination of the strategies are irrelevant. Similarly, CA125 was widely used and routinely performed in postmenopausal women with adnexal masses. A postmenopausal woman with a mass and a markedly elevated CA125 would have her surgery performed by a gynaecologic oncologist, regardless of the RMI, ROMA or IOTA results. Therefore, what is needed to actually assess the clinical value of these strategies is a prospective randomised study that evaluates their performance in patients who do not exhibit the obvious findings of malignancy, such as ascites, evidence of metastatic disease and elevated CA125, in which the management is dependent on the conclusion of the strategies. Then, outcomes, such as the need for second surgery, morbidity and overall survival, can be evaluated.

\section{Conclusions}

Apart from the strategies investigated in this study, many different methods or classification systems for predicting malignancy in pelvic masses detected on ultrasound were proposed, such as logistic regressions models [36] and, more recently, the simple rules risk 
model [37] and the ADNEX model [38]. There is increasing evidence supporting these new prediction models. Recently, ESGO/ISUOG/IOTA/ESGE published a consensus statement of pre-operative diagnosis of ovarian tumours. Subjective assessment by expert ultrasound has the best performance, but if such expertise is not available, the IOTA ADNEX model and IOTA simple rule risk model are recommended [39]. These methods require an online calculator or smartphone apps. The actual uptake of these newer assessment models into daily clinical practice is yet to be defined. Currently, the methods investigated in this study, namely IOTA simple rules, RMI and ROMA, were most widely used in clinical practice, mainly due to ease of use. Our study supported using IOTA simple rules as the first step in assessing a pelvic mass. For those with inconclusive results, an expert assessment would be superior to ROMA or RMI. If expertise is not available, assessment by ROMA or RMI are acceptable. The addition of IOTA to ROMA or RMI was better than ROMA or RMI alone. This would apply to both cancer centres and general units settings.

Author Contributions: Conceptualisation, S.F.N., J.L., M.M.Y.C., K.Y.T., H.Y.S.N., K.K.L.C.; Methodology, S.F.N., J.L., M.M.Y.C., K.Y.T., H.Y.S.N., K.K.L.C.; Formal Analysis, S.F.N., K.K.L.C.; Investigation, S.F.N., Y.K.C., K.M.C., T.W.L., G.S.T.K., M.M.Y.C., K.Y.T., V.Y.T.C., K.K.L.C.; Resources, S.F.N., Y.K.C., K.M.C., T.W.L., G.S.T.K., M.M.Y.C., K.Y.T., V.Y.T.C., K.K.L.C.; Data Curation, Y.K.C., K.M.C., T.W.L., K.K.L.C.; Writing-Original Draft Preparation, S.F.N., K.K.L.C.; Writing-Review and Editing, S.F.N., Y.K.C., K.M.C., T.W.L., J.L., G.S.T.K., M.M.Y.C., K.Y.T., V.Y.T.C., H.Y.S.N., K.K.L.C.; Visualisation, S.F.N., K.K.L.C.; Supervision, H.Y.S.N., K.K.L.C.; Project Administration, Y.K.C., K.M.C., T.W.L., V.Y.T.C., K.K.L.C.; Funding Acquisition, Y.K.C., K.M.C., M.M.Y.C., K.Y.T., V.Y.T.C., H.Y.S.N., K.K.L.C. All authors have read and agreed to the published version of the manuscript.

Funding: This research was funded by the Health and Medical Research Fund (HMRF), grant number 15161881.

Institutional Review Board Statement: The study was conducted according to the guidelines of the Declaration of Helsinki, and approved by the Institutional Review Board of the University of Hong Kong/Hospital Authority Hong Kong West Cluster (UW17-551, January 2018), the Research Ethics Committee (Kowloon Central/Kowloon East) (KC/KE-18-0014/ER-2, March 2018), and the Research Ethics Committee (Hong Kong East Cluster) (HKECREC-2018-004, February 2018).

Informed Consent Statement: Informed consent was obtained from all subjects involved in the study. Data Availability Statement: Not applicable.

Acknowledgments: We take this opportunity to extend our gratitude to Michelle KY Siu, Stephanie S Liu, Lesley SK Lau, Tina N Wei, and Esther TY Lui for their assistance in the sample collection and performing the CA125 and HE4 assays. We would also like to thank Helen Zhi and Lesley SK Lau for their help in the statistical analysis.

Conflicts of Interest: The authors declare no conflict of interest.

\section{References}

1. Engelen, M.J.; Kos, H.E.; Willemse, P.H.; Aalders, J.G.; de Vries, E.G.; Schaapveld, M.; Otter, R.; van der Zee, A.G. Surgery by consultant gynecologic oncologists improves survival in patients with ovarian carcinoma. Cancer 2006, 106, 589-598. [CrossRef] [PubMed]

2. Woo, Y.L.; Kyrgiou, M.; Bryant, A.; Everett, T.; Dickinson, H.O. Centralisation of services for gynaecological cancers-A Cochrane systematic review. Gynecol. Oncol. 2012, 126, 286-290. [CrossRef] [PubMed]

3. Meys, E.M.; Kaijser, J.; Kruitwagen, R.F.; Slangen, B.F.; Van Calster, B.; Aertgeerts, B.; Verbakel, J.Y.; Timmerman, D.; Van Gorp, T. Subjective assessment versus ultrasound models to diagnose ovarian cancer: A systematic review and meta-analysis. Eur. J. Cancer 2016, 58, 17-29. [CrossRef] [PubMed]

4. Van Calster, B.; Valentin, L.; Froyman, W.; Landolfo, C.; Ceusters, J.; Testa, A.C.; Wynants, L.; Sladkevicius, P.; Van Holsbeke, C.; Domali, E.; et al. Validation of models to diagnose ovarian cancer in patients managed surgically or conservatively: Multicentre cohort study. BMJ 2020, 370, m2614. [CrossRef] [PubMed]

5. Westwood, M.; Ramaekers, B.; Lang, S.; Grimm, S.; Deshpande, S.; de Kock, S.; Armstrong, N.; Joore, M.; Kleijnen, J. Risk scores to guide referral decisions for people with suspected ovarian cancer in secondary care: A systematic review and cost-effectiveness analysis. Health Technol. Assess. 2018, 22, 1-264. [CrossRef] 
6. Chacon, E.; Dasi, J.; Caballero, C.; Alcazar, J.L. Risk of Ovarian Malignancy Algorithm versus Risk Malignancy Index-I for Preoperative Assessment of Adnexal Masses: A Systematic Review and Meta-Analysis. Gynecol. Obs. Investig. 2019, 84, 591-598. [CrossRef]

7. Jacobs, I.; Oram, D.; Fairbanks, J.; Turner, J.; Frost, C.; Grudzinskas, J.G. A risk of malignancy index incorporating CA 125, ultrasound and menopausal status for the accurate preoperative diagnosis of ovarian cancer. Br. J. Obs. Gynaecol. 1990, 97, 922-929. [CrossRef]

8. Khoiwal, K.; Bahadur, A.; Kumari, R.; Bhattacharya, N.; Rao, S.; Chaturvedi, J. Assessment of Diagnostic Value of Serum Ca-125 and Risk of Malignancy Index Scoring in the Evaluation of Adnexal Masses. J. Midlif. Health 2019, 10, 192-196. [CrossRef]

9. Zhang, S.; Yu, S.; Hou, W.; Li, X.; Ning, C.; Wu, Y.; Zhang, F.; Jiao, Y.F.; Lee, L.T.O.; Sun, L. Diagnostic extended usefulness of RMI: Comparison of four risk of malignancy index in preoperative differentiation of borderline ovarian tumors and benign ovarian tumors. J. Ovarian Res. 2019, 12, 87. [CrossRef]

10. Royal College of Obstetricians \& Gynaecologists. Management of Suspected Ovarian Masses in Premenopausal Women. Greentop Guideline No. 62. Available online: https:/ / www.rcog.org.uk/globalassets/documents/guidelines/gtg_62.pdf (accessed on 2 January 2022).

11. Geomini, P.; Kruitwagen, R.; Bremer, G.L.; Cnossen, J.; Mol, B.W. The accuracy of risk scores in predicting ovarian malignancy: A systematic review. Obs. Gynecol. 2009, 113, 384-394. [CrossRef]

12. Zurawski, V.R., Jr.; Orjaseter, H.; Andersen, A.; Jellum, E. Elevated serum CA 125 levels prior to diagnosis of ovarian neoplasia: Relevance for early detection of ovarian cancer. Int. J. Cancer 1988, 42, 677-680. [CrossRef]

13. Hellstrom, I.; Raycraft, J.; Hayden-Ledbetter, M.; Ledbetter, J.A.; Schummer, M.; McIntosh, M.; Drescher, C.; Urban, N.; Hellstrom, K.E. The HE4 (WFDC2) protein is a biomarker for ovarian carcinoma. Cancer Res. 2003, 63, 3695-3700. [PubMed]

14. Moore, R.G.; Brown, A.K.; Miller, M.C.; Skates, S.; Allard, W.J.; Verch, T.; Steinhoff, M.; Messerlian, G.; DiSilvestro, P.; Granai, C.O.; et al. The use of multiple novel tumor biomarkers for the detection of ovarian carcinoma in patients with a pelvic mass. Gynecol. Oncol. 2008, 108, 402-408. [CrossRef]

15. Moore, R.G.; McMeekin, D.S.; Brown, A.K.; DiSilvestro, P.; Miller, M.C.; Allard, W.J.; Gajewski, W.; Kurman, R.; Bast, R.C., Jr.; Skates, S.J. A novel multiple marker bioassay utilizing HE4 and CA125 for the prediction of ovarian cancer in patients with a pelvic mass. Gynecol. Oncol. 2009, 112, 40-46. [CrossRef] [PubMed]

16. Van Gorp, T.; Cadron, I.; Despierre, E.; Daemen, A.; Leunen, K.; Amant, F.; Timmerman, D.; De Moor, B.; Vergote, I. HE4 and CA125 as a diagnostic test in ovarian cancer: Prospective validation of the Risk of Ovarian Malignancy Algorithm. Br. J. Cancer 2011, 104, 863-870. [CrossRef] [PubMed]

17. Chan, K.K.; Chen, C.A.; Nam, J.H.; Ochiai, K.; Wilailak, S.; Choon, A.T.; Sabaratnam, S.; Hebbar, S.; Sickan, J.; Schodin, B.A.; et al. The use of HE4 in the prediction of ovarian cancer in Asian women with a pelvic mass. Gynecol. Oncol. 2013, 128, 239-244. [CrossRef]

18. Wang, J.; Gao, J.; Yao, H.; Wu, Z.; Wang, M.; Qi, J. Diagnostic accuracy of serum HE4, CA125 and ROMA in patients with ovarian cancer: A meta-analysis. Tumour. Biol. 2014, 35, 6127-6138. [CrossRef] [PubMed]

19. Wilailak, S.; Chan, K.K.; Chen, C.A.; Nam, J.H.; Ochiai, K.; Aw, T.C.; Sabaratnam, S.; Hebbar, S.; Sickan, J.; Schodin, B.A.; et al. Distinguishing benign from malignant pelvic mass utilizing an algorithm with HE4, menopausal status, and ultrasound findings. J. Gynecol. Oncol. 2015, 26, 46-53. [CrossRef]

20. Timmerman, D.; Ameye, L.; Fischerova, D.; Epstein, E.; Melis, G.B.; Guerriero, S.; Van Holsbeke, C.; Savelli, L.; Fruscio, R.; Lissoni, A.A.; et al. Simple ultrasound rules to distinguish between benign and malignant adnexal masses before surgery: Prospective validation by IOTA group. BMJ 2010, 341, c6839. [CrossRef]

21. Testa, A.; Kaijser, J.; Wynants, L.; Fischerova, D.; Van Holsbeke, C.; Franchi, D.; Savelli, L.; Epstein, E.; Czekierdowski, A.; Guerriero, S.; et al. Strategies to diagnose ovarian cancer: New evidence from phase 3 of the multicentre international IOTA study. Br. J. Cancer 2014, 111, 680-688. [CrossRef]

22. Knafel, A.; Banas, T.; Nocun, A.; Wiechec, M.; Jach, R.; Ludwin, A.; Kabzinska-Turek, M.; Pietrus, M.; Pitynski, K. The Prospective External Validation of International Ovarian Tumor Analysis (IOTA) Simple Rules in the Hands of Level I and II Examiners Ultraschall Med. 2016, 37, 516-523. [CrossRef] [PubMed]

23. Nunes, N.; Ambler, G.; Foo, X.; Naftalin, J.; Widschwendter, M.; Jurkovic, D. Use of IOTA simple rules for diagnosis of ovarian cancer: Meta-analysis. Ultrasound Obs. Gynecol. 2014, 44, 503-514. [CrossRef] [PubMed]

24. Peces Rama, A.; Llanos Llanos, M.C.; Sanchez Ferrer, M.L.; Alcazar Zambrano, J.L.; Martinez Mendoza, A.; Nieto Diaz, A. Simple descriptors and simple rules of the International Ovarian Tumor Analysis (IOTA) Group: A prospective study of combined use for the description of adnexal masses. Eur. J. Obs. Gynecol. Reprod Biol. 2015, 195, 7-11. [CrossRef] [PubMed]

25. Ruiz de Gauna, B.; Rodriguez, D.; Olartecoechea, B.; Auba, M.; Jurado, M.; Gomez Roig, M.D.; Alcazar, J.L. Diagnostic performance of IOTA simple rules for adnexal masses classification: A comparison between two centers with different ovarian cancer prevalence. Eur. J. Obs. Gynecol. Reprod Biol. 2015, 191, 10-14. [CrossRef]

26. Sayasneh, A.; Wynants, L.; Preisler, J.; Kaijser, J.; Johnson, S.; Stalder, C.; Husicka, R.; Abdallah, Y.; Raslan, F.; Drought, A.; et al. Multicentre external validation of IOTA prediction models and RMI by operators with varied training. Br. J. Cancer 2013, 108, 2448-2454. [CrossRef]

27. Kaijser, J.; Sayasneh, A.; Van Hoorde, K.; Ghaem-Maghami, S.; Bourne, T.; Timmerman, D.; Van Calster, B. Presurgical diagnosis of adnexal tumours using mathematical models and scoring systems: A systematic review and meta-analysis. Hum. Reprod Update 2014, 20, 449-462. [CrossRef] 
28. Kaijser, J.; Van Gorp, T.; Smet, M.E.; Van Holsbeke, C.; Sayasneh, A.; Epstein, E.; Bourne, T.; Vergote, I.; Van Calster, B.; Timmerman, D. Are serum HE4 or ROMA scores useful to experienced examiners for improving characterization of adnexal masses after transvaginal ultrasonography? Ultrasound Obs. Gynecol. 2014, 43, 89-97. [CrossRef]

29. Hartman, C.A.; Juliato, C.R.; Sarian, L.O.; Toledo, M.C.; Jales, R.M.; Morais, S.S.; Pitta, D.D.; Marussi, E.F.; Derchain, S. Ultrasound criteria and CA 125 as predictive variables of ovarian cancer in women with adnexal tumors. Ultrasound Obstet. Gynecol. Off. J. Int. Soc. Ultrasound Obstet. Gynecol. 2012, 40, 360-366. [CrossRef]

30. Alcazar, J.L.; Pascual, M.A.; Olartecoechea, B.; Graupera, B.; Auba, M.; Ajossa, S.; Hereter, L.; Julve, R.; Gaston, B.; Peddes, C.; et al. IOTA simple rules for discriminating between benign and malignant adnexal masses: Prospective external validation. Ultrasound Obstet. Gynecol. Off. J. Int. Soc. Ultrasound Obstet. Gynecol. 2013, 42, 467-471. [CrossRef]

31. Knafel, A.N.; Nocun, A.; Banas, T.; Wiechec, M.; Jach, R.; Pietrus, M. Iota simple ultrasound-based rules: Why do we have inconclusive results? Int. J. Gynecol. Cancer 2013, 23, 155-156.

32. Piovano, E.; Cavallero, C.; Fuso, L.; Viora, E.; Ferrero, A.; Gregori, G.; Grillo, C.; Macchi, C.; Mengozzi, G.; Mitidieri, M.; et al Diagnostic accuracy and cost-effectiveness of different strategies to triage women with adnexal masses: A prospective study. Ultrasound Obstet. Gynecol. Off. J. Int. Soc. Ultrasound Obstet. Gynecol. 2017, 50, 395-403. [CrossRef]

33. Lycke, M.; Kristjansdottir, B.; Sundfeldt, K. A multicenter clinical trial validating the performance of HE4, CA125, risk of ovarian malignancy algorithm and risk of malignancy index. Gynecol. Oncol. 2018, 151, 159-165. [CrossRef] [PubMed]

34. Karlsen, M.A.; Sandhu, N.; Hogdall, C.; Christensen, I.J.; Nedergaard, L.; Lundvall, L.; Engelholm, S.A.; Pedersen, A.T.; Hartwell, D.; Lydolph, M.; et al. Evaluation of HE4, CA125, risk of ovarian malignancy algorithm (ROMA) and risk of malignancy index (RMI) as diagnostic tools of epithelial ovarian cancer in patients with a pelvic mass. Gynecol. Oncol. 2012, 127, 379-383. [CrossRef] [PubMed]

35. Education and Practical Standards Committee. Minimum training recommendations for the practice of medical ultrasound. Ultraschall Med. 2006, 27, 79-105. [CrossRef]

36. Timmerman, D.; Testa, A.C.; Bourne, T.; Ferrazzi, E.; Ameye, L.; Konstantinovic, M.L.; Van Calster, B.; Collins, W.P.; Vergote, I.; Van Huffel, S.; et al. Logistic regression model to distinguish between the benign and malignant adnexal mass before surgery: A multicenter study by the International Ovarian Tumor Analysis Group. J. Clin. Oncol. 2005, 23, 8794-8801. [CrossRef] [PubMed]

37. Timmerman, D.; Van Calster, B.; Testa, A.; Savelli, L.; Fischerova, D.; Froyman, W.; Wynants, L.; Van Holsbeke, C.; Epstein, E.; Franchi, D.; et al. Predicting the risk of malignancy in adnexal masses based on the Simple Rules from the International Ovarian Tumor Analysis group. Am. J. Obs. Gynecol. 2016, 214, 424-437. [CrossRef]

38. Van Calster, B.; Van Hoorde, K.; Valentin, L.; Testa, A.C.; Fischerova, D.; Van Holsbeke, C.; Savelli, L.; Franchi, D.; Epstein, E.; Kaijser, J.; et al. Evaluating the risk of ovarian cancer before surgery using the ADNEX model to differentiate between benign, borderline, early and advanced stage invasive, and secondary metastatic tumours: Prospective multicentre diagnostic study. BMJ 2014, 349, g5920. [CrossRef]

39. Timmerman, D.; Planchamp, F.; Bourne, T.; Landolfo, C.; du Bois, A.; Chiva, L.; Cibula, D.; Concin, N.; Fischerova, D.; Froyman, W.; et al. ESGO/ISUOG/IOTA/ESGE Consensus Statement on preoperative diagnosis of ovarian tumors. Ultrasound Obs. Gynecol. 2021, 58, 148-168. [CrossRef] 